\title{
Taxonomy and distribution of Andrographis longipedunculata (Acanthaceae)
}

\author{
G. Gnanasekaran ${ }^{1,2, *}$, K.V.C. Gosavi ${ }^{3}$, A.N. Chandore ${ }^{4}$, Mujaffar Shaikh $^{5}$ and G.V.S. Murthy ${ }^{1}$ \\ ${ }^{1}$ Botanical Survey of India, Southern Regional Centre, TNAU Campus, Lawley Road, Coimbatore - 641003, \\ Tamil Nadu, India. \\ 2Department of Botany, Madras Christian College (Autonomous), East Tambaram, Chennai - 600059, \\ Tamil Nadu, India. \\ ${ }^{3}$ Department of Botany, HPT Arts and RYK Science College, Nashik - 422005, Maharashtra, India. \\ ${ }^{4}$ Department of Botany, Abasaheb Marathe Arts and New Commerce, Science College, Rajapur - 416702, \\ Maharashtra, India. \\ ${ }^{5}$ Department of Botany, Government College, Bhikangaon, Khargone - 451331, Madhya Pradesh, India. \\ *E-mail: sekaranmcc@gmail.com
}

\begin{abstract}
Andrographis longipedunculata (Sreem.) L.H. Cramer (Acanthaceae), a lesser known endemic species of India is reported here for the first time from Myanmar and twelve more states of India. Also, illustrations, colour photographs, Scanning Electron Micrographs of pollen grains and seeds and a comparative table showing differences between its allied species, $A$. echioides are provided to facilitate accurate identification.
\end{abstract}

Keywords: Andrographis, Distribution, Indoneesiella, Myanmar, Taxonomy

\section{Introduction}

Sreemadhavan (1967) proposed a new genus, Neesiella Sreem. (nom. illeg.) to accommodate Andrographis echioides (L.) Nees (= Justicia echioides L.) and a new species, Neesiella longipedunculata Sreem. based on the collection [K. Subramanyam 4696] from Nagpur district in Maharashtra. He later realised that his Neesiella (1967) is a later homonym of Neesiella Schiffn. (1893) and therefore provided a substitute name, Indoneesiella Sreem. in 1968. However, Cramer (1996) reduced it to a subgenus of Andrographis Wall. ex Nees and proposed a necessary combination, $A$. longipedunculata (Sreem.) L.H. Cramer.

The present paper is a part of a systematic study on the genus Andrographis in India (Gnanasekaran, 2015), which is primarily based on the critical examination of fresh and herbarium specimens housed at BSD, BSI, BSJO, CAL, CALI, E, G, MH, P, RHT, SKU and SUK. The examination of specimens revealed that majority of $A$. longipedunculata specimens have been mistakenly identified as A. echioides (L.) Nees, an allied species and also showing an extended distribution from thirteen more states of India hitherto not recorded. Further, it is reported here as an new addition to the Flora of Myanmar for the first time. Thus, detailed taxonomic account along with illustration (Fig. 1), colour and Scanning Electron Micrographs of pollen grains and seeds (Fig. 2) and a comparative table showing differences between its allied species is provided here to facilitate accurate identification.

Andrographis longipedunculata (Sreem.) L.H. Cramer, Kew Bull. 51: 555. 1996; Karthik. et al., Fl. Pl. India 1: 3. 2009; U.L. Tiwari \& K. Ravik., Taprobanica 6: 132. 2014. Type: INDIA, Maharashtra, Nagpur district, Ambazeri tank, 317 m, 18.11.1957, K. Subramanyam 4696 (holo, MH00000796!; iso, MH00000797-MH00000802!).

Figs. 1,2

Neesiella longipedunculata Sreem., Phytologia 15: 271. 1967, nom. illeg.

Indoneesiella longipedunculata Sreem., Phytologia 16: 466. 1968; K.M. Matthew, Fl. Tamilnadu Carnatic 3: 1182. 1983; Kumari in A.N. Henry et al., Fl. Tamil Nadu 2: 150. 1987; Moulali in Pull. \& Moulali, Fl. Andhra Pradesh 2: 715. 1997; K.M. Matthew, Fl. Palni Hills: 940. 1999; S.S. Rani \& Nazeeruddin in Pull. et al., Fl. Eastern Ghats 4: 436. 2011; Vij. Sank. et al., Pl. Resources Tiruvannamalai Distr.: 473. 2012.

Erianthera longipedunculata (Sreem.) M.R. Almeida, Fl. Maharashtra 4A: 41. 2003.

Herb, to $80 \mathrm{~cm}$ high. Stems 4-angled, swollen at nodes, densely glandular-villous. Leaves sessile, 


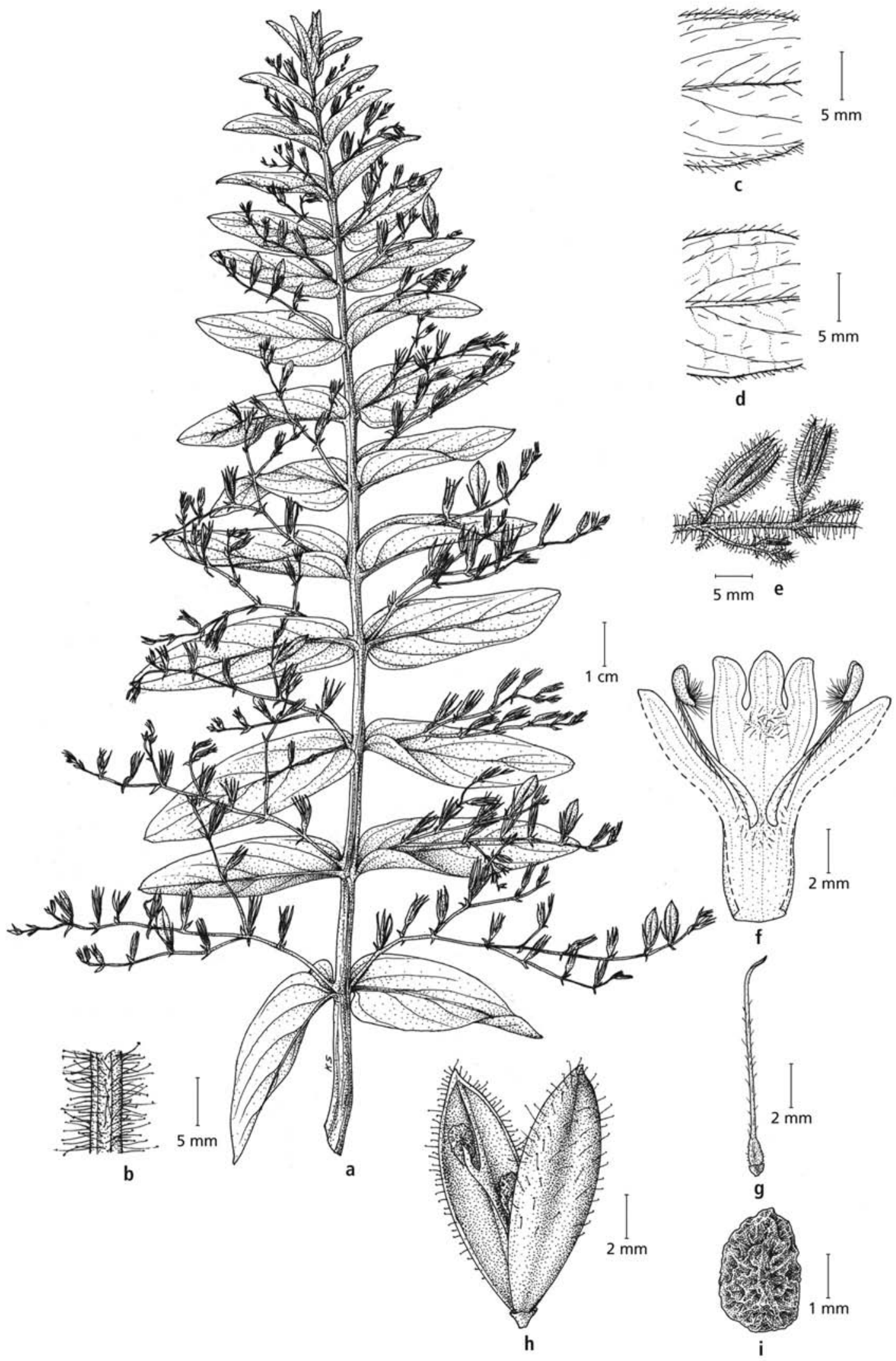

Fig. 1. Andrographis longipedunculata (Sreem.) L.H. Cramer: a. Habit; b. Close-up of indumentum on a portion of stem; c,d. Leaf surfaces: c. adaxial, d. abaxial; e. Close-up of indumentum on a portion of inflorescence; f. Corolla - Split-open; g. Pistil; h. Fruit - Split-open; i. Seed (a [s. coll. 9671, MH], b-i [J.V. Sudhakar 126914, MH]). 
ovate-lanceolate, $2-7.5 \times 0.5-2.5 \mathrm{~cm}$, base obtuse, acute or slightly cordate, margins entire, apex acute or obtuse; midrib conspicuous beneath; lateral veins 5-7 pairs, conspicuous beneath, strigulose intermixed with long glandular hairs on both surfaces. Inflorescence racemose, axillary, 4-10 cm long, often branched; axis 4-angled, densely glandular-pubescent; flowers distantly arranged (interstices $0.8-1.5 \mathrm{~cm}$ long), twisted at right angles to each other, one-sided; peduncles to $3 \mathrm{~cm}$ long, densely glandular-pubescent; pedicels indistinct, stout, 0-2 $\mathrm{mm}$, densely glandularpubescent. Bracts 2, subulate-lanceolate, 1.3-2.5 $\times \quad 0.2-0.3 \mathrm{~mm}$, apex acute, glandular-pilose. Bracteoles 2, linear, 0.5-0.8 $\times 0.05-0.15 \mathrm{~mm}$, apex acute to acuminate, glandular-pilose. Calyx 5-lobed; lobes linear, subequal, 5-8 $\times 0.3-0.5$ $\mathrm{mm}$, margins hairy, apex acute to subacuminate, strigulose inside, glandular-hairy outside. Corolla 2-lipped, $1-1.3 \mathrm{~cm}$ across, white; tube prominently ventricose, $6-7 \times 1.2-1.8 \mathrm{~mm}$, glandular-hirsute above, glabrous below; upper lip spathulateoblong, margins entire, minutely 2-lobed (c. 0.5 $\mathrm{mm}$ long) at apex, 3.8-4.2 $\times 2-2.8 \mathrm{~mm}$, glabrous inside, glandular-hirsute outside, 4-veined; lower lip 3-lobed, 4.5-5.5 × 3.5-4.3 mm, margins entire, apex acute or obtuse, glabrous inside except at centre of middle lobe, glandular-hirsute outside, dark purple-striped with yellow shade at centre; middle lobe narrowly ovate, $2-2.2 \times 1.5-1.7 \mathrm{~mm}$, hispid at centre, 3-veined; lateral lobes oblong, 1.2-1.4 mm across, 3-veined. Stamens 2, exserted, adnate to base of ventricose portion of corolla tube; filaments $5-7 \mathrm{~mm}$ long, distinctly dilated at base, retrorsely pilose above, glabrous below; anthers linear-oblong, $1.8-2.2 \times 0.3-0.5 \mathrm{~mm}$, woolly at base, deep purple. Ovary ellipsoid, $0.8-1$ $\times 0.5-0.7 \mathrm{~mm}$, glandular-hairy above, glabrous below, green; ovules 2 in each cell; style 8-10 mm long, sparsely antrorsely bristled-hairy, pinkish; stigma linear, green. Capsules ellipsoid, 8-10 $\times$ 3-4 mm, compressed at right angles to septum with a median longitudinal groove, apiculate, glabrous below, glandular-hairy towards apex, 4-seeded; seeds narrowly elliptic-obovoid, 3-3.2 $\times 1.8-2 \mathrm{~mm}$, base oblique, apex obtuse, very hard, lacunosus (deeply pitted), echinate, glabrous, yellowish brown.

\section{Flowering \& fruiting: July-March.}

Habitat: Plains, foothills, wastelands and barren ground; ready colonizer; occasional.

Distribution: India [Andhra Pradesh, Bihar, Diu and Daman, Goa, Gujarat, Haryana, Jammu and Kashmir, Jharkhand, Karnataka, Kerala, Madhya Pradesh, Maharashtra, Odisha, Punjab, Rajasthan, Tamil Nadu, Telangana, Uttar Pradesh and West Bengal] and Myanmar.

Conservation Status: Andrographis longipedunculata is widespread in the Indian states and is also represented by a large collection in the Indian herbaria. Therefore, it is evaluated here as of 'Least Concern [LC]' using the IUCN Red List Categories and Criteria version 3.1 (IUCN, 2017).

Etymology: The specific epithet 'longipedunculata' denotes the long (5 longi) peduncle of the inflorescence.

Inter-relationship: Andrographis longipedunculata is morphologically very similar to $A$. echioides and it can be distinguished from this species as given in Table 1.

Specimens examined: INDIA, India Orientalis, s.die, Roxburgh s.n. (E00435367, E00435368, images!); India Orientalis, s.die, V. Jacquemont 1534 (P03590506, P03590512, P03590512, images!); India Orientalis, s.die, R. Wight 2239 (P03590511, image!). Andhra Pradesh: Anantapur district, NS Right Canal, 125 m, 26.9.1985, V. Ramakrishnaiah 3558 (SKU); Danduvari palli, 356 m, 28.12.2009, B. Ravi Prasad Rao \& D. Veeranjanalu 35955 (SKU); Chittoor district, Chandragiri, 26.2.1914, s.coll. 10102 (MH); Cuddapah district, Palakonda R.F., 19.1.1987, D.A. Moulali 4682 (SKU); East Godavari district, Rampa hills, 26.9.1920, V. Narayanaswami 124 (CAL); Guntur district, Nagarjunakonda hills,

Table 1. Differentiating characters between Andrographis longipedunculata and A. echioides

\begin{tabular}{lll}
\hline Characters & A. longipedunculata & A. echioides \\
\hline Leaves & Often narrowly ovate-lanceolate & Oblong-linear, oblanceolate or elliptic \\
Inflorescences & Longer than leaves, to $10 \mathrm{~cm}$ long, & Shorter or almost equal to leaves, to \\
& much-branched; flowers distantly & $5 \mathrm{~cm}$ long, simple, rarely branched; \\
& arranged & flowers comparatively closely arranged \\
Peduncles & Up to $4 \mathrm{~cm}$ long & Less than $1.5 \mathrm{~cm}$ long \\
\hline
\end{tabular}



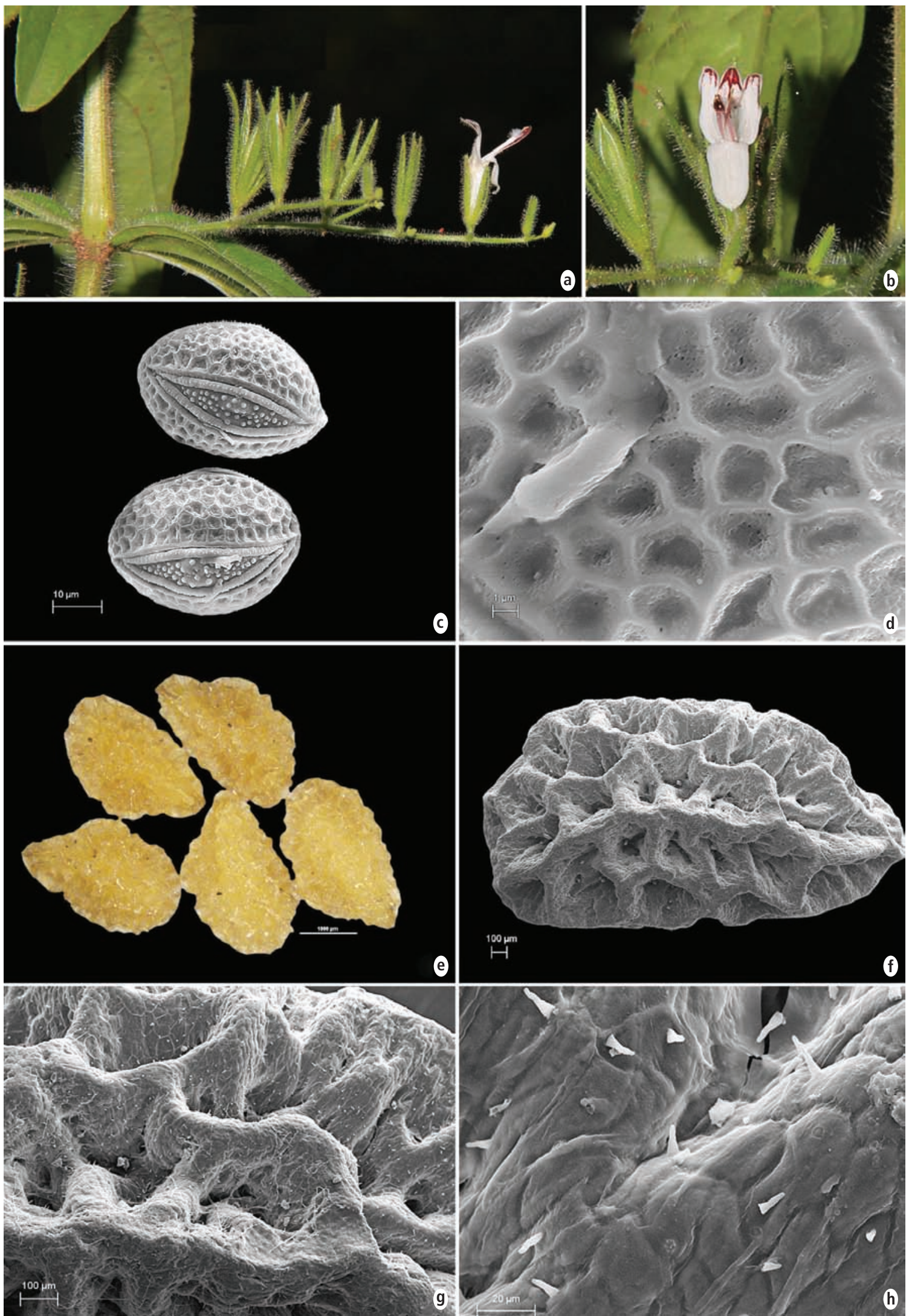

Fig. 2. Andrographis longipedunculata (Sreem.) L.H. Cramer: a. Flowering-twig; b. Flower; c,d. SEM micrograph of pollen; e-h. Optical and SEM micrographs of seeds. 
$500 \mathrm{ft}$ (c. $150 \mathrm{~m}), 26.11 .1961$, K. Thothathri 9762 (CAL); Mangalagiri, 21.10.1998, s.coll. 21616 (SKU); Krishna district, Kondapalli R.F., 13.9.1984, P. Venkanna 5306 (MH); Kurnool district, Pathikonda, s.die, A. Lakshmaiah 27894 (SKU); Erramalai hills, North Dhone R.F., 09.10.1983, R.R.V. Raju 1616 (SKU); Nellore district, Chintaldevi, 6.1.1920, s.coll. 16422 (MH); Balayapalli, 23.7.1995, P.M. Rajeswaramma 16457 (SKU); Prakasam district, Mussiri river side, Vengalapalem, 5.12.1983, R.K. Mohan 0338 (CAL); Vishakapatnam district, Anakapalli, 27.1.1916, s.coll. 12544 (MH); West Godavari district, Along railway tracks, Undi, Bhimavaram, 160 m, 15.10.2013, J.V. Sudhakar $126914(\mathrm{MH})$. Bihar: Santal Pargams, Trikut hills, 21.9.1992, J.K. Das 22297 (CAL); Gajgir, Vipulgiri, 13.9.2003, P. Pillai 32850 (CAL). Diu \& Daman: Diu island, 22.11.1962, T.A. Rao 1984 (CAL); Airport: NAGOA, 2.9.1964, K. Hemadri 102663 (CAL). Goa, North Goa, Near Pernem, 4.9.2015, Keshav Koli 413 (SUK). Gujarat: Ahmedabad, s.die, s.coll. s.n. (CAL); Ahmedabad, 24.10.1903, G.A. Gammie 163654 (BSI); Broach district, Gora opposite Narbada, 1.9.1967, R.D. Pateskar 110379 (BSI). Haryana: Damdama lake, 1.9.1999, S. Kumar 95237 (BSD). Jammu and Kashmir: Lauki Khad, 17.9.1996, B.P. Uniyal 92770 (BSD). Jharkhand: Chota Nagpore, Ramghur ghat, $1500 \mathrm{ft}$ (c. $450 \mathrm{~m}), 19.10 .1883$, C.B. Clarke 33903 (G00304825, image!); Chota Nagpore, Cheteu, tori, November 1880, J.S. Gamble 8721 (CAL); Hazaribagh district, Canary hills, 9.11.1964, K.C. Kanodia 1026 (CAL); Ranchi district, Duli forest, 20.11.1964, K.C. Kanodia 1317 (CAL); Basia, 21.2.1981, K.C. Malick \& al. 9775 (CAL); Santal Parganas district, Trikat hills, $1600 \mathrm{ft}($ c. $480 \mathrm{~m})$, 21.9.1992, J.K. Das E al. 22297 (CAL). Karnataka: Bellary district, Canara and Mysore, s.die, Law s.n. (CAL); Jogikallagudda R.F., 3.11.1975, N.P. Singh 141315 (BSI); Dharwar district, Dumbal, 20.9.1889, W.A. Talbot 1701 (CAL). Kerala: Kottayam district, Changanacherry, 28.4.1984, V.J. Antony 430 (CAL); Calicut University Campus, 5.5.1987, K. Laxmana 3732 (CALI). Madhya Pradesh: Indore district, Patalpani falls, 20.10.1962, A.S. Rao 83963 (CAL, BSI); Along rail track, Choral, 16.12.1961, A.S. Rao 79169 (BSI); Choral beride rail track, 21.10.1962, A.S. Rao 84045 (CAL, BSI); Jabalpur district, Water pool, Jabalpur, 26 December ?, G.T. Puri 30255 (BSI); Raisen district, Silwani, 29.8.1949, $V$. Narayanaswami 3599 (CAL); Sidhi district, Gandhigram, 22.10.1962, G. Panigrahi 5592 (CAL). Maharashtra: Akola district, Palodi, Mangarulpir, 2.9.1977, S.Y. Kamble 150360 (BSI); Aurangabad district, Near Ajanta caves, 6.11.1961, Rolla S. Rao 714007 (BSI); Buldhana district, Sultanpur, 23.9.1982, P.G. Diwakar 164085 (BSI); Chandrapur district, Kolsa Demi Road, 26.1.1972, B.M. Wadhwa 127308 (BSI); Khedsinghri Khurdrela, 16.10.1972, B.M. Wadhwa 130132 (BSI); Mohrhisam, 21.10.1972, B.M. Wadhwa 130237 (BSI); Moharh-Khahda Road, 18.8.1973, B.M. Wadhwa 137375 (BSI); Nandurbar district, Shahada, Susari dam, 18.9.2014, K.V.C. Gosavi 408 (SUK); Bhaler, 25.11.2015 K.V.C. Gosavi 409 (SUK); Nagpur district, Bandra Jungle, Pench National Park, 16.10.1984, B.D. Sharma 168707 (BSI); Nashik district, Chopda, 7.1.1957, S.K. Jain 11214 (BSI); Osmanabad district, Ghatangiri hill, 12.9.1964, K.P. Janardhanan 100656 (BSI); Pune district, Chakan, 15.10.1902, G.A. Gammie s.n. (BSI); Solapur district, Nannaj, 19.9.2010, J. Jayanthi E S.C. Yadav 198125 (BSI). Odisha: Bargarh district, Padarupur, 29.10.1959, G. Panigrahi 20525 (CAL); Amarakhol, 3.11.1959, G. Panigrahi 20770 (CAL). Punjab: Punjab, s.die, J.J. Thomson s.n. (CAL, P03590498, image!); Bhaeawal, 21.12.1904, L.D. Garade s.n. (BSI); Bhaeawal, 21.12.1904, G.A. Gammie s.n. (BSI); Bankhandi, 11.11.1959, T.A. Rao 10770 (BSD); Gurudaspur district, Pathankot, $500 \mathrm{~m}, 30.8 .1969$, U.C. Bhattacharyya 39446 (BSD); Hoshiarpur district, Mangunal, 27.8.1970, O.P. Mishra 41545 (BSD, image!); Shiwaliks district, Talwara, October 2000, Surendra Singh 97132 (BSD). Rajasthan: Erimpura, 1.10.1960, Rolla S. Rao 66774 (CAL); Dungarpur, 18.11.1957, J.A. Vasavada 20357 (BSI); Ajmer district, Nagpahar R.F., 1550 $\mathrm{ft}($ c. $460 \mathrm{~m}), 3.12 .1992$, R.P. Pandey \& P.M. Padhye 11250 (BSJO); Alwar district, Kanwari forest block, 23.10.2003,P.J. Parmar 2782 (CAL); Bhilwara district, Badnor, 20.9.1978, A.N. Singh 6260 (CAL); Bremer district, Sela forest Block, 375 m, 16.11.1981, R.P. Pandey 8047 (BSJO, CAL); Marwar district, Marwar, ? 1868, G. King s.n. (CAL). Tamil Nadu: Cuddalore district, Willingdon-Tholuthur, 100 m, 6.10.1978, P. Perumal 18183 (RHT); Dharmapuri district, Buddireddipatti, 19.12.1913, s.coll. 9671 (MH); Ramanathapuram district, Uttarakosamangai, $625 \mathrm{~m}, 1.1 .1988$, V. Balasubramaniam $1508(\mathrm{MH})$; Salem district, Mettur hills below dam, $170 \mathrm{~m}$, 17.12.1976, K.M. Matthew 5778 (RHT); Mettur, Gonur R.F., 350 m, 21.12.1976, K.M. Matthew \& V. Alphonse Raj 5995 (RHT); Chinnakalrayans, Thumbal R.F., 500 m, 9.1.1978, V.A. Amalraj 11052 (RHT); Thiruvannamalai district, Pakamalai R.F., 175 m, 21.1.1978, K. Ramamurthy 52876 (CAL, MH); Vilupuram district, Kallakurichi, Thottiam village tank, 150 m, 24.9.1978, N. Venugopal \& C. Manoharan 17709 (RHT); Vellimalai slopes (Kalrayan), 500 m, 21.10.1983, K. Ramamurthy 79627 (CAL, MH); Kallakuruchi tank land, 300 m, 23.10.1983, K. Ramamurthy 79679 (MH); Virudhunagar district, Sirumalai Pudupatti, 22.1.1979, N.C. Nair 61079 (CAL). Uttar Pradesh: Allahabad district, on the 
way to Moluli forest, 20.10.1964, G. Panigrahi 3604 (CAL); Kaligora, 15.12.1964, C.M. Arun 3997 (CAL); Banda district, Kalinpur, December 1902, A.S. Bell s.n. (CAL); Jansi district, Barwasagar, 8.3.1959, T.A. Rao 8342 (BSD, image!); Mirzapur, 9.2.1961, U.C. Bhattacharyya 13605 (BSD, image!). West Bengal: Midnapur district, Krishnager, 11.11.1997, T.K. Das E U.P. Samder s.n. (CAL); Chiligarh, 12.11.1997, T.K. Das \& U.P. Samader s.n. (CAL). MYANMAR, Upper Burma, 12.9.1890, Abdul Huk s.n. (CAL); Upper Burma, February 1892, Abdul Huk s.n. (CAL); Mundu, September 1902, Shaik Mokim 220 (CAL).

Note: Sreemadhavan (1967) indicated that the holotype of this species is housed at CAL. However, both holotype and isotypes are available only at $\mathrm{MH}$.

\section{Geographical Distribution}

The scrutiny of literature pertaining to the geographical distribution of the species revealed that it was hitherto reported to occur only in Maharashtra (Sreemadhavan, 1968), Tamil Nadu (Kumari, 1987), Andhra Pradesh (Moulali, 1997), Karnataka (Babu, 2011), Gujarat and Rajasthan (Tiwari \& Ravikumar, 2014), and Odisha (Murugan et al., 2017) from India. A thorough examination of specimens housed at various national and international herbaria in addition to the intensive field exploration in different parts of India resulted in ascertaining the occurrence of this lesser known endemic species from thirteen more states namely Bihar, Diu and Daman, Goa, Haryana, Jammu and Kashmir, Jharkhand, Kerala, Madhya Pradesh, Odisha, Punjab, Telangana, Uttar Pradesh and West Bengal from India. Further, while examining the specimens at CAL, senior author found a few specimens of this species collected from Upper Burma (Myanmar) were mistakenly identified as A. echioides. Therefore, the species is no longer can be considered as an endemic to India as it is reported here for the first time as a new record for Myanmar, showing an extended distribution of the species from outside of the Indian political boundaries.

\section{Acknowledgements}

Authors are grateful to Director, Botanical Survey of India (BSI), for facilities and to the Directors of BSD, BSI, BSJO, CAL, CALI, E, G, MH, P, RHT, SKU and SUK, for providing high resolution digital images or allowing authors to study the specimens. We also wish to thank Dr. W. Arisdason, Scientist, BSI, Kolkata, for his valuable suggestions and critical comments on the manuscript. Thanks are also due to Mr. K. Sivanandan, former Senior Artist, BSI, SRC, for preparing the line drawings. KVCG, ANC and MS are thankful to Science and Engineering Research Board (SERB), New Delhi, for financial assistance (File No.: SB/FT/ LS-130-/2012; SR/FT/LS-82/2012 and SB/YS/ LS-315/2013, respectively), under DST Fast Track Young Scientist Scheme.

\section{Literature Cited}

Babu, N.M.G. 2011. Flora and Ethnobotany of Chitradurga District, Karnataka. Ph.D. Thesis, Forest Research Institute University, Dehradun (unpublished).

Cramer, L.H. 1996. Notes on Sri Lankan Acanthaceae. Kew Bull. 51: 553-556.

Gnanasekaran, G. 2015. A systematic study on the genus Andrographis Wall. ex Nees (Acanthaceae) in India. Ph.D. Thesis, Bharathiar University, Coimbatore (unpublished).

IUCN Standard and Petitions Subcommittee, 2017. Guidelines for using IUCN Red List Categories and Criteria: Version 13. Downloaded from http://www.iucnredlist.org/document/ RedListGuidelines.pdf.

Kumari, G.R. 1987. Acanthaceae. In: Henry, A.N., Kumari, G.R. \& V. Chithra (eds.), Flora of Tamil Nadu. Ser. I. Analysis. Vol. 2. Botanical Survey of India, Coimbatore. pp. 138-162.

Moulali, D.A. 1997. Acanthaceae. In: Pullaiah, T. \& D.A. Moulali (eds.), Flora of Andhra Pradesh. Vol. 2. Scientific Publishers, Jodhpur. pp. 688-736.

Murugan, P., Kalidass, C. \& P.C. Panda 2017. Andrographis longipedunculata (Sreem.) L.H. Cramer (Acanthaceae) - An addition to the Flora of Odisha. Pl. Sci. Res. 39(1 \& 2): 82-83.

Sreemadhavan, C.P. 1967. Neesiella - A new genus of Acanthaceae. Phytologia 15: 270-271.

Sreemadhavan, C.P.1968. Indoneesiella-Asubstitute name in Acanthaceae. Phytologia 16: 466.

Tiwari, U. \& K. Ravikumar 2014. Andrographis longipedunculata (Sreem.) L.H. Cramer (Acanthaceae) from northwest India. Taprobanica 6: 132-134.

Received: 5.9.2016

Revised and Accepted: 22.12.2017 